\title{
INTERVIEW
}

For reprint orders, please contact: reprints@futuremedicine.com

\section{The role of surgery in low-grade gliomas: do timing and extent of resection matter?}

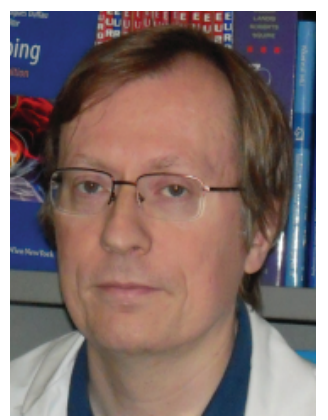

Hughes Duffau* speaks to Rochaine Wijayatunga, Commisioning Editor: Hugues Duffau is a Professor and Chairman of the Neurosurgery Department in the Montpellier University Medical Center and Head of the INSERM 1051 Team "plasticity of the central nervous system, human stem cells and glial tumors" at the Institute for Neurosciences of Montpellier (France). He is an expert in the awake cognitive neurosurgery of slow-growing brain tumors, such as low-grade gliomas, a routine which he has developed since 20 years. His fundamental approach is centered on the concepts of the brain connectomics and neuroplasticity, breaking with the traditional localizationist view of cerebral processing. For his innovative work in neurosurgery and neurosciences, he was awarded Doctor Honoris Causa several times, and he was the youngest recipient of the prestigious Herbert Olivecrona Award from the Karolinska Institute in Stockholm. He has written four textbooks and over 335 publications in international journals ranging from neurosurgery to fundamental neurosciences, including cognitive sciences and brain plasticity for a total of more than 20,800 citations and with an h-index of 77. He is a member of editorial boards of many journals (as brain and language, neurosurgery or neuro-oncology) and ad hoc reviewer for around 100 journals (over 900 reviews) including: New England Journal of Medicine, Lancet Oncology, Nature Medicine, Nature Reviews Neuroscience, Nature Reviews Neurology; Annals of Neurology, Brain, Cerebral Cortex, Trends in Cognitive Science, Current Biology, among others. He is a member of many societies: member of the French Academy of Medicine, member of the French Academy of Surgery, member of the World Academy of Neurological Surgery, member of the Young Neurosurgeons Award Committee of the World Federation of Neurosurgical Societies, member of the Scientific Committee of the European Association for Neuro-oncology, etc.

\section{Q What led to your interest in neurology \& in CNS oncology in particular?}

From the beginning of my medical studies, I was fascinated by the human brain, explaining why when I was resident, I had a parallel training in neurosurgery as well as in neurosciences. My goal was to better understand mechanisms underlying cerebral processing and to use this knowledge in order to help patients with brain tumors by improving not only their survival but also their quality of life. Therefore, very early on, I have tried to create links between cognitive neurosciences and neuro-oncology, especially by developing awake mapping in glioma surgery. This opened the window to the concepts of

\section{KEYWORDS}

- diffuse low-grade glioma • awake surgery $\bullet$ functional brain mapping - neuroplasticity $\bullet$ brain connectivity - quality of life 
neuroplasticity and brain connectome applied to the management of CNS tumor patients, by proposing a new paradigm, that is, early and maximal resection achieved according to individual functional boundaries - especially in diffuse low-grade gliomas.

\section{Q How have you seen your specific field of} research change since you began your career? As mentioned, my speciality is surgery for diffuse low-grade gliomas, usually involving young patients with an active life. So, this is the reason why I have a habit to speak mainly about quality of life because behavioral modifications may not be just indirect consequence of a disease like breast cancer for instance but also a possible direct consequence of the glioma on the brain itself and the perception we can have of health-related quality of life.

What I have seen in the past 20 years (because now I have approximately two decades of involvement in this field as a neurosurgeon), is first of all the increase of median survival. This morning (at ESMO 2016) we spoke about diffuse lowgrade gliomas. Ten years ago, median survival was between 6 and 7 years, now it is around approximately 15 years (from 10 to 20 years) so it means more or less one generation. Therefore, when I see a patient in front of me I know that he will have more or less one generation in order to do what he wants in his career (he will have time to progress in his professional life), but also maybe to have children, maybe to buy a house and so on. Thus, I think we can progress regarding this anticipation of what the patient would like to do for the next 10-20 years. Twenty years ago when I started my career, it was not possible to have this kind of discussion about tumoral chronic disease and quality of life individually defined for a glioma patient (according to his job/hobby/needs, etc.).

\section{Q As the head of the INSERM 1051 team} 'plasticity of the CNS, human stem cells \& glial tumors'. What are your main roles? Why was this team set up initially?

Neuroplasticity is something which was underestimated for many decades and even centuries. I mean, not only in CNS tumor, but also whatever the neurological disease is - speaking about stroke, multiple sclerosis and so on. Now, we have started to understand that not only does this plasticity exist in children but it also exists in adults. Second, we can more or less control this plasticity by avoiding to disrupt the 'connectome' speaking about CNS - I mean we have some limitations of plasticity related to the subcortical connectivity underpinning networking process of the brain.

We can also use the potential of functional reshaping of these networks in order to increase the quality of life of the patient by inducing neuroplasticity, thanks to the surgery itself, the treatments, antiepileptic drugs for instance (because it is always better to avoid having seizures for cerebral remapping), along with postoperative cognitive rehabilitation. Being able to push the mechanism for brain plasticity will also open the door for re-treatment of the patient, for instance to do a second or third surgery with an increase in the extent of resection in comparison with the first surgery while not against the quality of life. In other words, we are evolving to individualized management - real precision medicine - not just related to the genome but also the individual brain 'connectome'. So, you see that neuroplasticity is really a synapse, if I can say, between oncology and cognitive neuroscience.

In my research lab, my role is typically to make this synapse concrete in practice, by investigating mechanisms subserving neuroplasticity in patients who undergo awake surgery for a diffuse glioma, by studying interactions between the natural history of the tumoral disease and the CNS reaction (brain reorganization), and by applying this knowledge to tailor new dynamic and multistage therapeutic strategies at the individual level.

Q Can you tell our readers a little about the awake cognitive neurosurgery of slow-growing brain tumors that you have developed?

First of all, we know that there is no one eloquent area in the brain is dedicated to one function, for instance Broca's area is not the only area of language, this is just a part of a widely distributed network which can eventually be removed without any permanent aphasia. In other words, localizationism does not exist in CNS, this is only a dogma. On the other hand, we cannot say a priori before going to the operating room (OR) that this area is not crucial for function, because even though the patient would probably not be aphasic or hemiplegic following resection, he can have nonetheless, some problems regarding more subtle functions, as the cognitive function and 
emotional process. We spoke about the fact that the patient should be able to anticipate what he would like to do for the next 10-20 years and it is much more complex rather than to avoid language and motor deficits - even if this is of course also crucial. Indeed, you can remove the right temporal lobe for instance, but in spite of the fact that the patient will be able to speak fine after surgery, it does not mean that the patient will resume a perfect familial, social and professional life. In this state of mind, we developed new neuropsychological tests to assess neurocognition and behavior in glioma patients before going into the OR - first of all by stating if the patient is well before surgery. According to these results, beyond the fact that we should awake the patient in all cases throughout the resection, we will select the tasks for intraoperative mapping on the basis of the presurgical neuropsychological evaluation - that is, not a priori based on anatomical considerations only but based on functional considerations. As in my presentation this morning, it is possible now into the OR not only to map movement but also the control of movement, language, but also (nonverbal) semantics, cognition (as attention or working memory) but also emotion and social interaction - and everything useful to preserve the quality of life while optimizing the extent of resection. For instance, recently, I mapped the networks involved in empathy in a psychiatrist because he told me before surgery, that he absolutely wanted to be able to continue to deal with his patients, and just to speak and to move or to have a high-level of cognition is not enough because he needed the ability to mentalize and have empathy to continue to be a good psychiatrist. So, you see that we are doing a kind of 'mapping a-la-carte' - designed for each patient individually - according to his/her quality of life - that they should define before surgery.

Q You have achieved much in your career so far including being awarded the Doctor Honoris Causa many times \& you were also the youngest recipient of the Herbert Olivecrona award from Karolinska Institute in Stockholm. What would you say has been your most proud moment to date?

Each time I meet patients and their family telling me that they are happy, because they continue to enjoy a normal life 5, 10, 15 years following surgery.
Q You presented on "the role of surgery in low-grade gliomas: do timing \& extent of resection matter?" at ESMO 2016. Can you briefly tell our readers about your presentation?

The main message of my presentation is that it is impossible to take care of glioma patients if one is focusing only on the tumor without any considerations with regard to the brain itself. In other words, the aim is not to increase the overall survival whatever the price to pay concerning the quality of life. Both should be improved, by achieving early safe maximal resection using awake mapping, by preserving the individual connectome and by guiding neuroplasticity as we discussed above. We should be more ambitious by optimizing both survival and brain functions in glioma patients: the dilemma is solved, on the condition that one adapts the management at the individual level without applying a 'standard protocol' based upon the sole histomolecular profile of the tumor. In summary, in our surgical experience based on hundreds of low-grade glioma patients with almost 20 years of follow-up, the concept of 'functional surgical neuro-oncology' led to a median survival around 15 years with less than $1 \%$ of severe permanent deficit. Therefore, surgery should be considered in a more systematic way at diagnosis in all low-grade glioma patients, both for oncological and functional purposes, because radical resection is able to change dramatically the history of this tumor by preventing malignant transformation: so, the wait and watch attitude should be definitely abandoned.

Q What made you so convinced that "we did not have to unquestioningly accept the classical model of the brain," \& how did this lead you to suggest surgery of 'still-conscious patients with brain tumors'?

Just based on observations 20 years ago, the brain was accepted as rigid at least in adult patients. So, it meant that functions were static with no possibility to reorganise. Although, I learned that when I was a student, the first patients I saw in front of me were patients with these very extensive tumors within so-called eloquent areas according to the classical model of brain processing (as Broca's area, Wernicke's area or Rolandic area). However, these patients told me they 'felt well', so I thought that either this patient does not exist or the model is wrong. Of course, I decided to fight against the model and now, I have accumulated, 
as you have seen, 100s and 100s of patients with very extensive resection even in 'critical regions' according to the old view of CNS organization, and who are well. So, it means that I am considered not only as a neurosurgeon or surgical neurooncologist, but as a neuroscientist. Indeed, I have a parallel career in the small world of cognitive neurosciences, allowing me not only to improve survival and quality of life of glioma patients, but also to propose new dynamic models of cerebral processing based upon the observations made in these patients - for example, revisited connectomal model underlying movement control, language or mentalizing.

Q A recent paper you co-authored looked at the oncological, functional \& methodological difference between pediatric \& adult populations with regard to awake surgery. Can you tell our readers briefly what the main conclusions of your study were?

First, that the tumors are not the same in children and adults. This is the reason why I also said when speaking about a possible program of screening in the general population (see later), that we would like to understand the origin of the tumor and to see when this tumor is occurring because it is exceptional to see a child with low-grade glioma who finally became adult with anaplastic transformation of the tumor. In fact, most gliomas in children are well-delineated tumors (as pilocytic astrocytoma) while diffuse gliomas are more common in adults. Second, the management is different too because in children, if I simplify, you could have a benign tumor, you remove it and the patient can be cured (even though you should be careful of course about quality of life too, as the child has to continue to learn). Alternatively, you could have a very aggressive tumor, as a malignant glioma within the brainstem, and then surgery by itself is not a therapeutic option - it is much more a question to ask medical oncologists. Third, regarding surgical point of view, you imagine that you cannot awake a child that is too young (before 10 years old) in the OR and to ask him/her to help us to perform intraoperative brain mapping. You can do that approximately from 10 years old and it means that before this age we have to think differently (oncologically, functionally and surgically speaking) compared with supratentorial diffuse glioma in young adults.

\section{Can you talk a little about any specific research projects you have gong on currently?}

As mentioned, we have started to understand that one subfunction (movement, language, visuo-spatial processing and so forth) is not underpinned by one specific brain area, but by specific subcircuit in a networking view of CNS organization. The goal is now to investigate the interaction between these subnetworks, in order to provide new insights into human behavior. This is crucial in glioma patients because tumoral cells migrate along white matter tracts, thus limiting brain plastic potential, and eliciting cognitive or emotional troubles due to a disruption within the whole functional network(s). Thus, objective neuropsychological examinations should be performed in a systematic manner before and after each treatment in glioma patients. In addition, longitudinal functional neuroimaging studies combined with repeated cognitive assessments represent a unique opportunity to explore noninvasively the dynamic redistribution of cerebral sub-networks and their ecological consequences: such a knowledge will elaborate original therapeutic strategies adapted to each glioma patients over the years.

\section{Q What is/are the biggest challenge(s) to} research in the field of brain cancer research? Is it too risky to challenge the norm/standard approach?

One of the main challenges is to understand the origins of glioma in order to treat earlier, and to avoid the invasion of the brain connectome by the tumoral cells - that prevents complete surgical resection and that may lead to cognitive disorders. If a tumor is diagnosed when it is smaller and when it does not yet invade the subcortical connectivity too extensively, it may be possible to achieve a 'supratotal' resection, that is, to surgically remove a margin around the signal abnormality on FLAIR-weighted MRI. Indeed, in diffuse gliomas, in essence, tumoral cells exist several millimeters to centimeters beyond the lesion visible on neuroimaging. In this subgroup of supramarginal resection (achieved according to functional limits), we demonstrated that the rate of malignant transformation was almost nil, so with a very significant impact on the overall survival in patients leading a normal life (especially with no seizures) - and usually with no oncological adjuvant treatment. As a consequence, with the 
aim of increasing the chance to perform supracomplete resection, we have recently proposed a policy of screening in the general population.

\section{Q How do you hope this specific field in} brain tumor research will develop over the next 5-10 years?

First of all I would like to see more individualized multistage therapeutic strategies. Today, everyone is claiming that we are doing precision medicine but this is not true. We are applying protocol based upon most of the time just a few parameters, for instance molecular biology and that is it - and it is not enough. So, my dream is really to think that we can anticipate what we will do when we are seeing the patient for the first time, to explain to him and his family that he will live with a chronic disease and that we will not decide to incorporate him in this protocol, a priori based on very few parameters but that we will re-evaluate the individualized strategy through his life according to regular clinical, neuropsychological and radiological follow-up ad vitam aeternam.

The second point is to better understand the individual interactions between the brain and the tumor in clinical practice because I know that this synapse between CNS dynamics and glioma growth does not exist today. I am sure that today in this room when I spoke about brain processing $90 \%$ of people did not understand the message about neuroplasticity and cerebral connectomics. To me, it is crazy because they are managing patients with brain tumors without knowing the CNS. In my opinion, today the first end point should be the quality of life because we can radically change the median survival. But it is not possible to 'loop-the-loop' and make this link if we were not at least partly neuroscientists. This is my ambition now to have a more integrated view between the knowledge of the reaction of the magic CNS, when this kind of slow-growing tumor is treated. Such a philosophy will lead to the principle of what I like to call the 'onco-functional balance' which should be determined by the patient himself in order to guide the best therapeutic sequence according to his needs (which are not the same in a 20-year-old vs 50-year-old patient, or in a pianist vs a mathematician, and so forth.).

\section{Q Any closing remarks for our readers?}

To sum up, to treat low-grade glioma patients is not conceivable not only without understanding the natural course of the tumor at the individual level (volume and location of the glioma, study of growth rate on serial MRI, histomolecular criteria), but also without investigating the dynamic organization of CNS processing for each patient, and thus, the interaction between the disease, the host and the recursive and multimodal therapeutic strategy adapted over years. This strong relationship between cognitive neurosciences and CNS oncology should lead to the new concept of 'preventive and personalized functional neuro-oncology' breaking with the old dogma of 'wait and see' attitude in diffuse low-grade glioma, with the goal to significantly improve both the quantity and quality of life (including sensorimotor, language, cognitive and emotional functions) by tailoring individual management on the basis of the tumor behavior and brain plastic potential regularly re-evaluated ad vitam aeternam.

\section{Disclaimer}

The opinions expressed in this interview are those of the interviewees and do not necessarily reflect the views of Future Medicine Ltd.

\section{Financial \& competing interests disclosure}

The author has no relevant affliations or financial involvement with any organization or entity with a financial interest in or financial conflict with the subject matter or materials discussed in the manuscript. This includes employment, consultancies, honoraria, stock ownership or options, expert testimony, grants or patents received or pending, or royalties.

No writing assistance was utilized in the production of this manuscript. 